\title{
A Comparative Assessment of Floating and Submerged Sensor Network Deployments for Monitoring Underwater Sediment Transport Processes
}

\author{
Archie J. Watt, Carlene E.-A. Campbell, Stephen Hole, Ian Wells, Michael R. Phillips \\ School of Applied Computing, University of Wales Trinity Saint David, Swansea, UK \\ Email: archie.watt@uwtsd.ac.uk, carlene.campbell@uwtsd.ac.uk, stephen.hole@uwtsd.ac.uk, \\ ian.wells@uwtsd.ac.uk, mike.phillips@uwtsd.ac.uk
}

Received 18 April 2016; accepted 19 May 2016; published 26 May 2016

\begin{abstract}
Wireless Sensor Networks (WSNs) are a pioneering technology in many environmental monitoring applications owing to their ability to be deployed for long periods of time in locations that cannot be reached manually. One such use-case is the monitoring of underwater sediment transport, a process that plays a significant role in coastal erosion. Previous examples of WSNs deployed for this purpose have been in the form of underwater sensor networks (UWSNs), which have a number of shortcomings from both a practical and technical viewpoint. As such, this paper provides a comparative assessment of UWSNs and an alternative deployment approach of floating echosounding sensor networks for the purpose of monitoring underwater sediment transport.
\end{abstract}

\section{Keywords}

Wireless Sensor Networks, Environmental Monitoring, Underwater, Coastal Erosion

\section{Introduction}

Beaches are of great economic importance through a number of different industries, but most significantly travel and tourism. Travel and tourism is the world's largest industry, of which beaches are a key element [1]. Processes such as erosion that have a detrimental impact on a beach over time are a serious threat to beach tourism and by extension the national economy. The problem of coastal erosion is one that is becoming increasingly common and destructive worldwide as a result of rising sea levels. The collection of data to understand this process has to date been largely centered upon surveying and measuring exposed coastal areas such as beaches. Whilst this makes it possible to quantify the effects of coastal erosion, the inability to measure what is occurring beneath the surface of the sea means that only a partial view of coastal erosion is gained due to the lack of this important data. As such, a means to measure these underwater processes is highly desirable, as it would result in a more complete and accurate understanding of coastal erosion, and assist coastal engineers in more effective 
planning of coastal defense strategies.

The submerged beach profile is generally modeled by describing two zones, namely the near-shore zone and offshore zone. High volumes of sediment movement activity occur in the near-shore zone, whilst the offshore zone is largely inactive in this respect. These two zones are divided by a contour which is known as the depth of closure [2], as shown in Figure 1. Being able to measure the depth of closure would enable the gathering of valuable data relating to the dynamics of sediment movement on the seabed, and by extension make it possible to obtain more accurate measurements of coastal erosion.

Wireless has been an increasingly popular area of both academic and commercial interest as a result of the growing transition from wired to wireless communications. Wireless Sensor Networks (WSNs) [3]-[5] are an emerging technology that have rapidly become one of the fastest-growing areas in the communications industry due to the unique applications for which they can be utilized. In terms of monitoring the environment, they have particular advantages as they can be deployed in locations which cannot be reached manually. Their deployment underwater makes them an enabling technology with the potential of resolving the above issue by being able to collect data on underwater processes related to coastal erosion.

Underwater Sensor Networks (UWSNs) are typically submerged in the body of water in which they are operating and use acoustic waves as a transmission medium (sometimes known as hydro-acoustics), which is the most reliable and robust medium for underwater transmissions, despite suffering from such issues as extremely low bandwidth, and vulnerability to adverse effects caused by conditions such as turbidity, ambient noise, salinity, and pressure gradients [6]. Electromagnetics (EM) and optical communications are two alternative mediums, and although both have been proven to work in underwater deployments, they both suffer from limited range, and, in the case of the former, electromagnetic interference (EMI). Optical communications, despite far greater bandwidth capacities, it is only a realistic approach in very clear water, being a light based medium. Irrespective of the communication medium that is used, there are further problems relating to deployment, maintenance, and cost associated with UWSNs. As such, it is worthwhile to evaluate the merits of different types of WSN deployment. To this end, the focus of this paper is upon a comparative assessment of submerged pressure sensors and echo sounding floating WSN deployments for the purpose of monitoring sediment transport on the seabed.

This paper is structured as follows: sections 2 and 3 provide respective overviews of underwater and floating WSNs. Section 4 comparatively discusses these two types of WSN in the context of some of the common problems faced. Finally, section 5 presents the conclusions drawn.

\section{Underwater Sensor Networks}

Water covers around $75 \%$ of the earth's surface, much of which remains largely unexplored. UWSNs are an

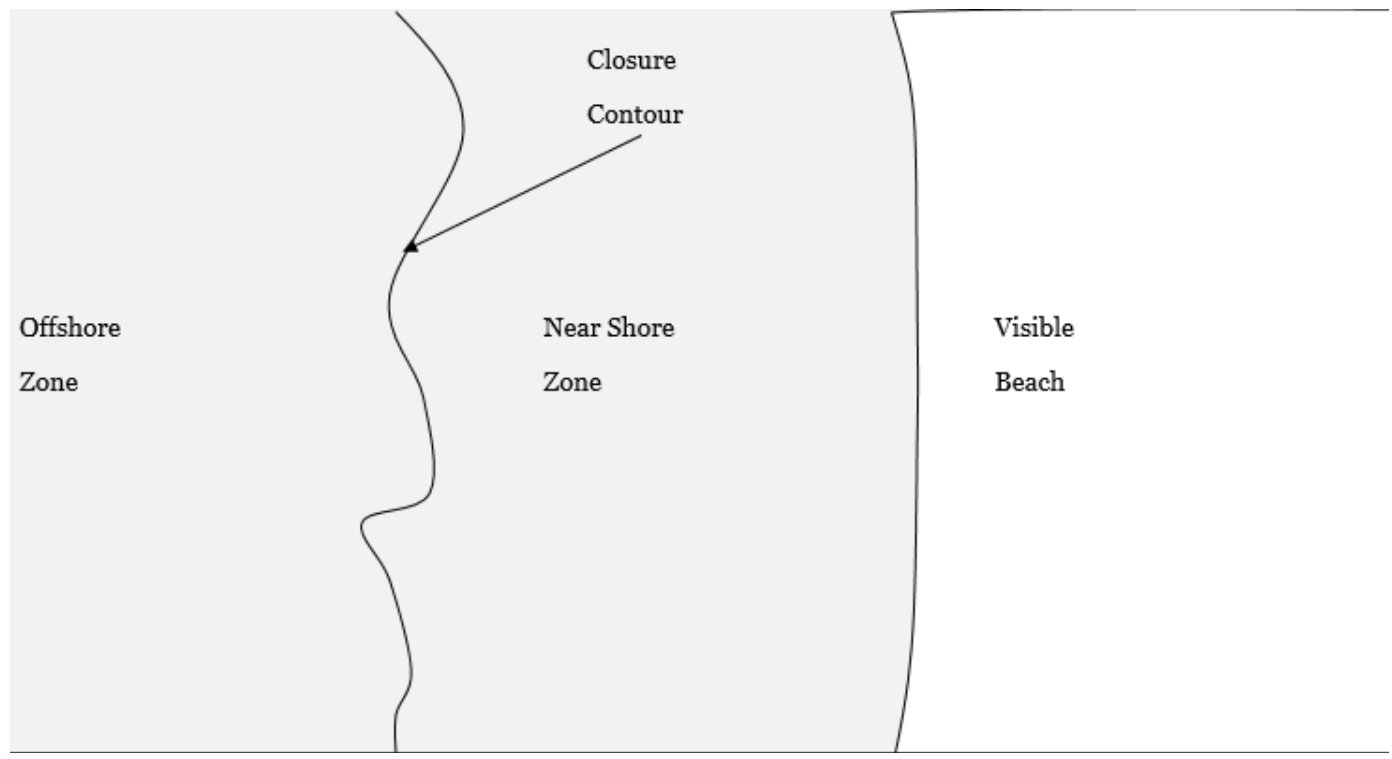

Figure 1. Depth of closure. 
enabling technology in terms of gathering data from the ocean, as they can be deployed in locations that cannot be reached or monitored long-term manually. An UWSN, illustrated in Figure 2 is a collection of autonomous sensor nodes which are spatially distributed underwater to collect data on various different parameters, which, depending on the application may include water quality, pressure, and temperature. In common with most WSNs, the nodes in the network transmit their collected data to a gateway (often called the "sink" node), which then transmits the data to its final destination for analysis. UWSNs typically use acoustic waves as a communication medium, which, despite low bandwidth, have long transmission ranges underwater and therefore make it possible for a single network to collect data from large areas.

Research in the area of underwater communications has been underway for at least half a century, with one of the first underwater communication devices being the underwater phone that was developed by the US Navy following World War II [8]. During the last decade, this type of communication has been receiving continually increasing interest due to the many scientific, military and commercial applications it has the potential to make a reality. These range from environmental monitoring, the study of marine life, and tactical surveillance.

\section{Echo Sounding Sensor Networks}

The term "sounding" is used to refer to all types of depth measurement, including earlier methods that, despite the name, did not utilize actual sound. The use of sounding lines to measure water depth dates back as far as Ancient Greek civilisations, and is achieved by using a rope with a weight attached that is lowered over the side of a ship. When the weight reaches the bottom the line would slacken and the depth at that particular time could then be marked at the surface. This method continued to be used throughout the twentieth century, however, the development of echosounders has now made sounding lines largely obsolete. An echo sounding sensor makes use of sonar technology to derive the height of a water column by sending a sound pulse which reflects ("echoes") off the seabed. The depth of the body of water is calculated based upon measurements of how long it takes the sound wave to reach the seabed and return. By correlating the change in depth measurement with tide level measurements, the change in sediment depth can be measured.

This approach is commonly known as echosounding, and is mathematically formulated as:

$$
\text { Distance }=\mathrm{t} /(2 \times \mathrm{s})
$$

Equation (1). Echosounding formula.

Where $t$ is the length of time the sound pulse takes to return, and $s$ is the speed at which sound travels through

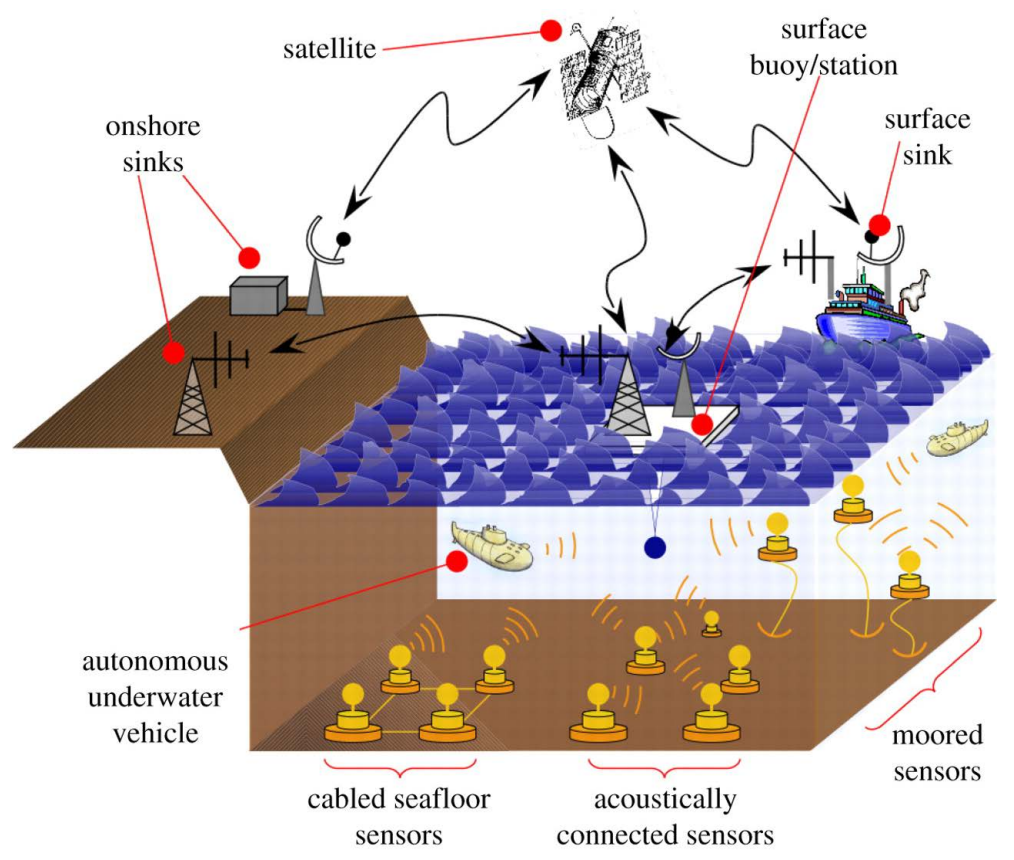

Figure 2. Underwater acoustic sensor networks [7]. 
water, which is approximately 1500 metres/second. This is generally used as the standard sound speed, though in the event that greater accuracy is required it may be necessary to deploy a sound velocity probe.

\section{Comparisons}

\subsection{Cost}

Sensor nodes in terrestrial WSNs are expected to become increasingly inexpensive. However, UWSNs require more complex transceivers that can function underwater as well as advanced hardware protection capable of withstanding the conditions of the underwater environment, such as pressure, extremely low temperatures and salinity levels. Additionally, the processing capabilities of sensor nodes may need to be more advanced; the underwater communication channel is likely to be intermittent meaning that it may be necessary for sensor nodes to perform data caching. This increases the cost of the devices as well as their power requirements.

Floating WSNs, whilst naturally requiring their hardware to be protected against water, need this to a much lesser extent, due to the absence of extreme pressure levels on the surface. Additionally, since communication occurs on the surface it is not necessary for nodes to communicate underwater, reducing the complexity of the transceivers and the processing requirements of the sensor nodes, and therefore the overall deployment and maintenance costs.

\subsection{Deployment and Maintenance}

Deployment of a UWSN is likely to be an intensive process; the sensor nodes are likely to be heavy and bulky due to the necessary hardware protection. Additionally, in order to ensure more accurate pressure measurements, the angle of the sensor node on the seabed would need to be observed, likely requiring the use of divers to ensure that the nodes are correctly positioned. However, the underwater environment can cause the node to move, which could potentially reduce the validity of the measurements. In terms of maintenance and reliability, once an UWSN is deployed it is virtually impossible to carry out any maintenance without retrieving the device from the seabed, meaning the device will be out of operation during this process. Finally, despite hardware protection, the pressure levels at the bottom of the sea are such that water damage is still possible.

These problems are largely absent with floating WSN deployments, due to their lesser water protection requirements. Additionally, they can be retrieved easily for maintenance purposes, and the ability to recharge batteries using solar cells and/or small wind turbines improves their longevity.

\subsection{Communication}

The three possible underwater communication technologies, acoustics, RF, and optical, have all have been proven to work underwater, but none of them can be classed as the one optimal medium for UWSNs. A WSN deployed for the same purpose of monitoring sediment transport is presented in [9]. Whilst demonstrating that RF communication is possible underwater, its use of very low frequencies resulted in a data rate of only 100 bps, which would not be sufficient for many UWSN applications, especially if the system needed to collect and transmit data on a regular basis.

A floating WSN does not need to communicate underwater; following data collection it can transmit readings to the sink node using wireless technologies such as ZigBee or 6LoWPAN, enabling the data communication to avoid issues such as extreme bandwidth limitations and propagation delays.

\subsection{Power Supply}

Energy efficiency is a problem prevalent in all battery-operated WSNs. However, this problem worsens when the WSN is deployed in a location where batteries cannot be easily replaced or recharged; such is the case with UWSNs. Additionally, the power requirements for underwater communications are greater, as more complex digital signal processing (DSP) has to be performed by the receivers in order to compensate for the channel impairments [7].

By communicating on the surface, floating WSNs not only reduce the communication power requirements, they also ease the process of replenishing sensor node battery power, either through battery replacements or by charging the battery using small solar panels or wind turbines on the surface. 


\subsection{Localization}

Localization refers to determining the location of the sensor node, which, for many applications is crucial in order for the collected data to be meaningful, and can also be important for such tasks as routing and node tracking [10]. Localization is achieved with ease in terrestrial sensor networks due to the availability of the Global Positioning System (GPS). However, the limited propagation of radio waves through water means that GPS will not work with UWSNs. Even if the sensor nodes are fixed in position it can be difficult to rely on the location, as the harshness of the underwater environment means that can still be moved around. As a result, localization techniques for UWSNs is an active research topic; a recent review of several proposed solutions is provided in [11].

Floating WSNs do not suffer from this problem; they communicate on the surface of the water and therefore can work with GPS easily. Additionally, the nodes can be tethered to a buoy so that their location can be visibly observed.

\subsection{Security}

Security is an important concern in any computer network, though generally more in those that use wireless communication, since no physical connection is required for an attacker to gain access. Whilst the attacks that a WSN is vulnerable to are much the same as any other wireless network, the problems are often exacerbated by the broader problem of energy limitations; the more complex a security protocol the more processing work a sensor node will need to do. As a result, it may be necessary to make a compromise between security and energy efficiency, though which takes priority will likely depend on the specific application of the WSN; if sensitive data is being collected, it will require encryption to prevent it being read in the event it was compromised.

Although security would be a concern regardless of the type of deployment, the increased power requirements of UWSNs mean that less power is available to perform the necessary processing to meet security requirements, potentially making them more vulnerable to being compromised. A floating deployment can make use of existing wireless networking protocols such as ZigBee and 6LoWPAN, which provides the facilities for carrying out secure communications [12]. Additionally, the reduced power requirements and the comparative ease of replacing and/or recharging batteries make more processing capability available to meet security requirements.

\subsection{Summary}

Table 1 summarizes this the comparisons discussed in this section.

\section{Conclusions}

This paper has assessed two approaches to deploying a sensor network for monitoring the movement of sediments on the seabed, from which it is suggested that the dominant means of deploying WSNs for this purpose to date is inefficient both practically and technically. Therefore, floating echo-sounding WSNs were investigated as an alternate approach, which showed considerable promise when compared to WSNs that are deployed underwater.

Table 1. Comparison of submerged and floating WSN deployments.

\begin{tabular}{|c|c|c|}
\hline Criteria & Underwater Sensor Networks & Floating Sensor Networks \\
\hline Cost & $\begin{array}{l}\text {-Complex node design } \\
\text {-Advanced hardware protection }\end{array}$ & $\begin{array}{l}\text { Less complexity required in transceiver design } \\
\text { and node processing }\end{array}$ \\
\hline $\begin{array}{l}\text { Deployment/ } \\
\text { Maintenance }\end{array}$ & $\begin{array}{l}\text {-Heavy and bulky due to hardware protection } \\
\text {-Difficult to maintain once deployed }\end{array}$ & $\begin{array}{l}\text { Relatively easy both to deploy and retrieve for } \\
\text { maintenance purposes. }\end{array}$ \\
\hline Medium & No optimal communication medium & $\begin{array}{l}\text {-No underwater communication necessary } \\
\text {-ZigBee, 6LoWPAN can be used }\end{array}$ \\
\hline Power Supply & $\begin{array}{l}\text {-Complex DSP resulting in higher power } \\
\text { requirements } \\
\text {-Difficult to replenish power }\end{array}$ & $\begin{array}{l}\text {-Reduced communication power requirements } \\
\text {-Easier to replace/recharge power supply }\end{array}$ \\
\hline Localization & No firmly established method. & $\begin{array}{l}\text { Can be used with GPS to accurately establish the } \\
\text { node's position. }\end{array}$ \\
\hline Security & $\begin{array}{l}\text { Increased power requirements, less power } \\
\text { available for security protocols. }\end{array}$ & $\begin{array}{l}\text { Can take advantage of establish standards that } \\
\text { consider security, such as ZigBee/6LoWPAN }\end{array}$ \\
\hline
\end{tabular}


Whilst this approach would not work for all UWSN applications, for the particular use-case of monitoring underwater sediment transport it appears to be a superior approach when compared to deploying sensors on the seabed.

Additional research is required to test this approach, and as such our future work will focus on designing and evaluating a small echosounding WSN in a lab environment, which will enable the feasibility of this method to be tested. Following this, more extensive field trials can be undertaken in order to test the system in a real-world environment.

\section{References}

[1] Houston, J. (2013) The Economic Value of Beaches-A 2013 Update. Shore \& Beach, 81, 3-10.

[2] Phillips, M. and Williams, A. (2007) Depth of Closure and Shoreline Indicators: Empirical Formulae for Beach Management. J. Coast. Res., 232, 487-500. http://dx.doi.org/10.2112/05-0593.1

[3] Karl, H. and Willig, A. (2005) Protocols and Architectures for Wireless Sensor Networks. John Wiley and Sons. http://dx.doi.org/10.1002/0470095121

[4] Campbell, C.E.-A., Shah, I.A. and Loo, K.-K. (2010) Medium Access Control and Transport Protocol for Wireless Sensor Networks: An Overview. Int. J. Appl. Res. Inf. Technol. Comput., 1, 79-92. http://dx.doi.org/10.5958/j.0975-8070.1.1.005

[5] Corke, P., Wark, T., Jurdak, R., Hu, W., Valencia, P. and Moore, D. (2010) Environmental Wireless Sensor Networks. Proc. IEEE, 98, 1903-1917. http://dx.doi.org/10.1109/JPROC.2010.2068530

[6] Che, X., Wells, I., Dickers, G., Kear, P. and Gong, X. (2010) Re-Evaluation of RF Electromagnetic Communication in Underwater Sensor Networks. IEEE Commun. Mag., 143-151. http://dx.doi.org/10.1109/MCOM.2010.5673085

[7] Heidemann, J., Stojanovic, M. and Zorzi, M. (2012) Underwater Sensor Networks: Applications, Advances and Challenges. Philos. Trans. R. Soc. A Math. Phys. Eng. Sci., 370, 158-175. http://dx.doi.org/10.1098/rsta.2011.0214

[8] Headrick, R. and Freitag, L. (2009) Growth of Underwater Communication Technology in the U.S. Navy. IEEE Commun. Mag., 47, 80-82. http://dx.doi.org/10.1109/MCOM.2009.4752681

[9] Che, X., Wells, I., Kear, P., Dickers, G., Gong, X.G.X. and Rhodes, M. (2009) A Static Multi-Hop Underwater Wireless Sensor Network Using RF Electromagnetic Communications. 29th IEEE Int. Conf. Distrib. Comput. Syst. Work., 2009. http://dx.doi.org/10.1109/icdcsw.2009.36

[10] Erol-Kantarci, M., Mouftah, H.T. and Oktug, S. (2010) Localization Techniques for Underwater Acoustic Sensor Networks. IEEE Commun. Mag., 48, 152-158. http://dx.doi.org/10.1109/MCOM.2010.5673086

[11] Nageswararao, K. and Prasan, U.D. (2012) A Survey on Underwater Sensor Networks Localization Techniques. Int. J. Eng. Res. Dev., 4, 1-6.

[12] Dini, G. and Tiloca, M. (2010) Considerations on Security in ZigBee Networks. SUTC 2010-2010 IEEE Int. Conf. Sens. Networks, Ubiquitous, Trust. Comput. UMC 2010-2010 IEEE Int. Work. Ubiquitous Mob. Comput., 58-65. http://dx.doi.org/10.1109/sutc.2010.15 